Experience of Caregivers Supporting a Patient Through Voluntarily Stopping Eating and Drinking (VSED)

Revised for Journal of Palliative Medicine May 2020 


\section{Experience of Caregivers Supporting a Patient Through Voluntarily Stopping Eating and Drinking}

(VSED)

\section{Abstract \\ Background}

Voluntarily stopping eating and drinking (VSED) is an ongoing voluntary choice to forego food and hydration in an effort to hasten death. Ongoing caregiving is necessary as patients become weak and lose focus as a result of dehydration, but little is known about the process of supporting a patient through VSED.

\section{Objective}

To explore the experiences of caregivers who supported a patient through VSED.

\section{Methods}

Qualitative study with thematic analysis of transcripts of semi-structured interviews with 24 U.S. caregivers for 20 individuals who had attempted VSED

\section{Results}

Analysis produced four themes: 1) Caregivers believe that VSED is the best death available to the patient. 2) Caregivers act as advocates and worry that the patient's goals will be challenged by healthcare professionals, the community, or legal authorities; obtaining support from hospice is an important way to legitimize VSED. 3) Through the VSED process itself, caregivers carry the responsibility for the patient's success as the patient becomes weaker and loses focus. 4) Because there is no social script to guide the VSED process, caregivers choose what roles to play during VSED, such as focusing on physical care or being emotionally present as the patient's spouse or child.

\section{Conclusions}


Caregivers face unique challenges in helping patients undertake VSED. Many are uncertain about whether they will receive support from clinicians or the community. Support from health professionals may improve caregiver confidence and reduce worry. 


\section{Background}

Voluntarily stopping eating and drinking (VSED) is an ongoing voluntary choice to forego food and hydration to hasten death. Unlike medical aid in dying (AID), VSED does not require lethal medication or a physician's order, although symptom management is recommended and caregiving is necessary, both for physical symptoms and as patients lose focus because of dehydration (1).

Symptoms related to VSED include thirst, progressive weakness, and sometimes delirium (2). Patients become sleepier over time and may enter a coma several days before death. Death may be expected in 10-14 days depending on fluid intake $(3,4)$.

Symptom management includes easing dry mouth, help with toileting and hygiene, and managing anxiety, agitation or delirium (5). Patients may ask for water in dehydration-related confusion, and caregivers may need to remind patients that drinking fluids may slow the dying process $(2,5)$.

A recent Swiss study estimated that $1.7 \%$ of nursing home residents die by VSED (6). Dutch studies have produced estimates of $0.4 \%$ to 1.75 of annual deaths (4). Results from a 2001 survey mailed to Oregon hospice nurses found, $41 \%$ reported having cared for a patient who attempted or completed VSED (3). Patients' average age was $74 ; 54 \%$ were women; and cancer was the most frequent diagnosis (60\%), followed by neurologic (23\%) or cardiovascular disease (16\%).

No studies have specifically addressed the roles or experiences of caregivers assisting a patient with VSED.

\section{Methods}


This qualitative study included single, in-depth, semi-structured interviews with adults who had supported a patient undertaking VSED. Inductive thematic analysis in Nvivo 12.0 (QSR International) followed Braun and Clarke's six steps: familiarization, generating initial codes, building potential themes, testing themes against data, defining themes, and producing the report. (7) The PI, a former medical journalist and community educator on end of life issues, conducted all interviews and analysis and used a reflexive journal after each interview and during coding to explore possible bias, such as potentially over-empathizing with certain caregivers, such as only children caring for parents $(8,9)$. The interview guide was developed following consultation with two VSED caregivers and included prompts related to the patient's personality and health; discussion of VSED and planning; initiation and progression of VSED; death; and aftermath (See Box). 


\section{Guiding Questions and Probes}

- Tell me a little about [patient].

○ Personality, background, family dynamics

- Illness and its effects

- How did the idea of stopping eating and drinking come up?

- Caregiver reactions

- Other options considered

- Other reactions from family, friends

- Making the choice of VSED/supporting VSED

- What happened after [patient] made that decision?

- Planning - medical or legal preparation, logistics

- Caregiver thoughts and reactions

- Caregiver perceptions of own role

- Worries or concerns

- How did the process actually start/what happened next (early, middle, late stages)?

- What changes caregiver noticed, reactions

- Patient's needs, caregiver's role

- Communication with patient and others

- Role of other caregivers and hospice

- Were there problems you had to solve? What happened?

- Caregiver's thoughts and feelings in that time

$\circ$ What made things easier or harder

- What happened when [patient] died?

- Feelings at the time

- Was it the death patient wanted, why or why not

- Caregivers' actions post-death

- Caregiver's life in days/weeks following death

- Communicating with others about death, including whether VSED was mentioned

- Do you feel like you can talk about the way [patient] died?

- Summing up

- What was most important about going through VSED with patient

o What went well, and what parts were hardest

- What does the caregiver wish had gone differently

- What could others learn from this experience?

- What else is important to know about your experience? 
Braun and Clarke suggest 10-20 interviews as a target for medium-sized thematic analysis studies (8).

Participants (Table 1: Inclusion and Exclusion Criteria) were recruited through conference flyers and emails describing the study sent to clinicians, researchers, end-of-life rights advocates, and journalists to forward to potential participants. All potential participants who contacted the PI received a description of the research project and provided informed consent. Participants could withdraw for up to one month after the interview.

Interviews (range 45-105 minutes) were conducted by phone (12), in person (3), or video conference (8), and were audio recorded and transcribed verbatim. The study protocol and recruitment materials were approved by [university] Research Ethics Committee.

\section{Results}

A total of 24 caregivers completed interviews. Caregivers cared for 20 patients who undertook VSED, with 19 resulting deaths. The median time until death was 10 days (range $2-28$ days). Patients choosing VSED fit one of three categories: a diagnosis of early dementia; progressive, terminal illness; or being done with living. The latter category consisted primarily of elderly, frail individuals with chronic health problems or a recent change in health that reduced independence. One patient halted her VSED effort after three weeks because hired aides and nursing staff at her assisted living facility did not provide the necessary support for VSED, such as medication for anxiety or non-fluid forms of relief from thirst. In four cases, two caregivers were interviewed about the same patient. VSED attempts took place in 10 states between 2009-18, with a median of five years between the death and the interview.

Patient and caregiver characteristics are described in Table 2. All caregivers were white and had at least some college education; half had advanced degrees. Caregivers' median age was 61 (range 26-86) at time of VSED; patients' median age at death was 80 (range 43-94). 
Reflexive thematic analysis asserts that themes are constructed both from the data and the researcher's interpretation, with a goal of generating themes that can clearly be drawn from the data (8). Inductive analysis of transcripts initially generated more than 100 codes related to caregivers' experiences with VSED. Subsequent rounds of analysis collated these codes into subthemes representing concrete aspects of caregiver experience, and finally four major themes (Table 3) described below.

\section{VSED is the best death available to the patient}

Caregivers contrasted VSED with other trajectories of living and dying available to the patient and concluded that VSED was the best death available to the patient. For most caregivers, belief in the right of individuals to choose their mode of dying was a prerequisite for their own ability to come to terms with the patient's choice, support the patient, and evaluate for themselves the options the patient might consider.

In cases of early dementia, the patient and caregiver knew that aid in dying was not legally available, rendering VSED the best available means to meet the patient's wishes.

"She knew it was not an option, to give her medication to make her die. She wasn't terminal by any stretch of the imagination. So I don't think it was the ideal for her, but she was glad to have an exit strategy. She was glad it went well, she was happy along the way." -son caring for mother with early dementia

Caregivers of patients with dementia and progressive illness consistently said that the alternative death expected by the patient through disease progression was "horrible," "unacceptable," or "pretty sad", with low quality of life or heavy symptom burden. VSED, by contrast, was "peaceful" and "a very good 
death." Most caregivers of patients who were tired of living found meaning in the patient being able to exert control in their life at the end, particularly when poor health had limited their independence.

\section{Risk and legitimacy}

Once committed to supporting the patient's choice to pursue VSED, caregivers often acted as patients' agents to obtain whatever medical, social, or other support seemed necessary. While caregivers understood and supported patients' goals, many expressed some sense of risk that external parties might object to VSED or try to stop it. To mediate that risk, caregivers sought markers that the patient's choice was legitimate.

Nearly all patients and caregivers actively sought hospice care for VSED, but caregivers worried that either the referring physician or hospice might decline to provide care. Patients with cancer were already enrolled in hospice. Other patients were not eligible based on Medicare criteria, but caregivers hoped initiating VSED would qualify the patient. Ultimately, $85 \%$ of patients were enrolled in hospice care. Several patients lived in areas where the only hospice was Catholic-run and declined to support VSED.

For caregivers, hospice provided not only symptom management but legitimacy. Several were conscious of the potential that others in the community may view VSED as a form of suicide or elder abuse. Some pointed to the experience of Phyllis Shacter (10), whose book about her husband's death through VSED includes an incident in which Adult Protective Services visited the home because of an anonymous report of elder abuse.

“I didn't like want to tell the neighbor. I didn't want someone to feel like, 'Well, you can't do that! Stop!' And we had hospice around, so that lent some legitimacy to it. I can only imagine if there was someone who didn't have hospice who just was at home with their loved one waiting 
for them to die. I bet that would be really hard." -daughter caring for mother with progressive genetic condition

Some caregivers waited until after the patient's death before telling anyone outside the family what had happened. Perception of risk of social stigma appeared to be more common for caregivers caring for patients who did not outwardly appear to be plausibly near death, particularly patients early in dementia whose symptoms might not be noticed in casual conversation.

\section{Carrying responsibility for the patient's success}

VSED is characterized by a gradual decline in patients' physical function and mental clarity during dehydration. Many caregivers noted that as patients' capacity decreased, the caregivers took on the full weight of responsibility for ensuring that patients succeeded in VSED.

Caregivers consistently listed independence as among patients' dominant personality traits, but at some point in the VSED process patients handed off responsibility for the outcome to caregivers. In dementia, handoffs of power and responsibility occurred early because patients were able to state and follow through on their desire to hasten death through VSED but lacked the executive functioning to participate in the planning or preparation. Caregivers for patients with cognitive decline described a window of opportunity when quality of life had diminished enough to motivate the patient to start VSED but the patient still retained the capacity to choose not to drink. The patient's diminishing function became a threat against success of VSED.

Caregivers monitored for threats to the patient's success, often arising directly from the patient's deteriorating condition. As dehydration progressed, patients became physically weaker, increasing the chance that they could fall and injure themselves seriously enough to require medical attention. Caregivers assumed that emergency clinicians would hydrate the patient, or that a fracture would result in inpatient care in a rehabilitation setting that would not support VSED. 
"For the first few days until she really became totally bedridden, ... I'd be sleeping and I'd wake up finding her halfway out of bed, heading for the bathroom. ... So my fear was if she falls and breaks her hip, then the whole plan is derailed and she'll have to go to the hospital and then how will they treat her in terms of nutrition and fluids? ... So I was very vigilant during that whole period, which is exhausting." -husband caring for wife with progressive genetic condition

Caregivers took steps to minimize patients' exposure to tempting food or drink. Many avoided cooking in the house or had friends bring meals that could be eaten when the patient was asleep. Thirst was a bigger challenge than hunger. Some patients complained of thirst, particularly as advanced dehydration fogged their executive function. Across the cases, caregivers arrived at a consistent practical and ethical approach to patients' request for fluids. When a patient asked for liquids, caregiver responses generally included:

- A reminder that not drinking was the patient's choice and part of reaching their goal of dying

- Reassurance that the caregiver would give them something to drink if they really wanted it

- Acknowledgment that any fluids would slow down the dying process

- An offer for alternative relief, such as a glycerin swab or mist spray

\section{There is no script}

Most caregivers had not previously cared for someone dying, much less someone actively choosing to die. The relative certainty of death created a sense of finitude, but some caregivers were unsure how to facilitate meaning for the patient or create it for themselves. Combined with the improvised logistics and plans caregivers made to support what they thought the patient would need, the unique circumstances of VSED left some caregivers feeling that there was no script for the VSED process. 
Caregivers entered the VSED process with the certainty that if all went to plan, the patient would die within a matter of days to weeks. Caregivers simultaneously carried their hopes for the patient's successful death and their own anticipatory grief. Caregivers also perceived that time as both too long for one person to manage the entirety of care without becoming exhausted, but a very short time in which to say goodbye or seek closure.

Many caregivers felt they lacked the skill or endurance to attend to the patient's physical needs during VSED, and most hired aides to help. But some caregivers with clinical expertise chose to forego providing physical care in order to focus on being emotionally present with the patient. This emotional focus was something many caregivers felt they could provide uniquely as a spouse or child, but also as a gift to themselves, to make the most of the last days with the patient. One described attempting to care for her partner at home before eventually securing a bed for him at a residential hospice:

"I probably had 8-9 days of tracking, 'Shit, when's the last time we gave you morphine? Do you want an ice chip? Do you not want an ice chip? Do you want a washcloth?' I mean, 20 days of that, I couldn't have done it. Thank god I didn't have to. ... The idea that there were real live nurses [at the hospice] who were helping was just a huge load off my mind. I could do my emotional work which was enormous without trying to figure out who can bathe Willie." partner caring for patient with cancer

Caregivers took on the role of closure facilitator, inviting close friends and family to visit and say goodbye or managing encounters per the patient's wishes. Some helped the patient with drafting an obituary or communicating plans for financial assets.

Many caregivers pushed their own feelings about loss aside to focus on helping the patient succeed in dying. Some avoided sharing their own feelings of grief so as not to weaken the patient's resolve. The 
intensity of caring for a patient through the early and middle stages of VSED meant that some caregivers confronted the emotional weight of the patient's impending death only toward the end.

"That span of time was difficult because I was the one trying to make the family all coherent, trying to do everything I could do for her, trying to get quality time, trying to bring food into the house so the family could eat, running around like crazy. It was sort of like I had to bury all the feelings I was having until later." -husband caring for wife with ALS

Some caregivers and patients had plans for passing the time with old photos or favorite music and movies, but, some patients with cognitive decline were unable to focus or were agitated in the early days of VSED, leaving some caregivers frustrated because hoped-for closure was no longer feasible. Other caregivers perceived that patients were withdrawing into themselves, either because of their illness or to focus on the dying process, leaving the caregiver unsure how to insert their own needs for closure into the time available.

\section{Discussion}

The four themes presented above demonstrate caregivers' commitment to helping patients complete VSED despite uncertainty, possible risk, and their own grief. In VSED, caregiving is an immersive, consuming process to which caregivers commit entirely. As patients' dependence increases, caregivers assume responsibility for their success. Despite lack of previous experience, many VSED caregivers anticipate the magnitude of the task and take steps to ensure that the process not only is successful for the patient but one the caregivers themselves can endure and find meaning in. Uncertainty about whether VSED will be perceived as a legitimate choice by health professionals or organizations or the community increases some caregivers' sense of risk before and during caregiving. Caregivers' thoughts about external reactions to VSED suggest that on some level caregivers carry an awareness about who in society can die without censure: the visibly ill, the frail and elderly. Patients intent on VSED flouted those 
conventions by pursuing death on their own terms.

\section{Strengths and limitations}

The scant research conducted on VSED has taken the form of clinician surveys $(3,11,12$ which suggest the prevalence of VSED in various countries and the reasons patients undertake it, but the true population of VSED caregivers is unknown. A strength of this research is that it accessed a diffuse population and investigate their experiences for the first time. A further strength is that caregivers' characterizations of patients' reasons for pursuing VSED are consistent with U.S. and Dutch findings in terms of somatic and psychosocial reasons for wanting to die $(3,11,13)$. This suggests that the study has captured some of the breadth of types of VSED cases. The demographics of participants - white and highly educated - may not reflect the true diversity of individuals undertaking VSED or VSED caregiving; however, in the United States patients who pursue AID are mostly white and have high education attainment (14).

Most caregivers in this study viewed VSED positively. Individuals with negative experiences may not have wanted to be interviewed; thus this study may not have captured the true breadth of VSED caregiver experience. In this study, patients had died several months to 10 years prior to the interview, and caregivers' perceptions of events may have changed over time (15).

Implications for practice

These findings represent several contributions to scholarship on hastened death. Understanding the experience of VSED caregivers helps distinguish it among other forms of hastened death and sheds light on how patients and families choose and undertake intentional dying. The experience of caregivers of 
people who chose VSED not because of illness but because they were done with living also challenges assumptions that VSED is pursued only when aid in dying (AID) is unavailable.

Public and political discourse around hastened death is evolving rapidly in the United States, as one in five Americans now lives in a jurisdiction where AID is legal. For those who live elsewhere or who fall outside the strict qualifications for AID, VSED may be an option of interest. Identifying similarities and differences between caregiver experiences and needs in VSED and at end of life can guide care planning for hospice and palliative professionals, as well as primary care clinicians, who may be the first to learn of patients' intentions.

\section{Future research needs}

Future research could explore the motivations of individuals seeking VSED themselves or capture the experience of an individual undergoing VSED. A study with multiple palliative care or hospice programs could systematically document VSED-related inquiries and attempts in a population. Future research could study physician and hospice staff beliefs and hospice policies around VSED.

No competing financial interests exist.

\section{References}


1. Quill TE, Ganzini L, Truog RD, Pope TM. Voluntarily Stopping Eating and Drinking Among Patients With Serious Advanced IIIness-Clinical, Ethical, and Legal Aspects. JAMA Internal Medicine. 2018;178(1):123-7.

2. Wax JW, An AW, Kosier N, Quill TE. Voluntary Stopping Eating and Drinking. J Am Geriatr Soc. 2018;66(3):441-5.

3. Ganzini L, Goy ER, Miller LL, et al. Nurses' Experiences with Hospice Patients Who Refuse Food and Fluids to Hasten Death. The New England Journal of Medicine. 2003;349(4):359-65.

4. Royal Dutch Medical Association. Caring for people who consciously choose not to eat and drink so as to hasten the end of life. Utrecht; 2014.

5. Schwarz J. Exploring the option of voluntarily stopping eating and drinking within the context of a suffering patient's request for a hastened death. Journal of Palliative Medicine. 2007;10(6):1288.

6. Stangle S, Schnepp W, Buche D, Fringer A. Long-term care nurses' attitudes and the incidence of voluntary stopping of eating and drinking: A cross-sectional study. J Adv Nurs. 2020;76(2):526-34.

7. Braun V, Clarke V. Using thematic analysis in psychology. Qualitative Research in Psychology. 2006;3(2):77-101.

8. Braun V, Clarke V. Successful Qualitative Research: A Practical Guide for Beginners: London: SAGE Publications; 2013.

9. Hennink MM. Qualitative research methods. Bailey A, Hutter I, editors. London: SAGE; 2010.

10. Shacter P. Choosing to Die: A Personal Story. United States of America: Phyllis Shacter; 2017.

11. Bolt EE, Hagens M, Willems D, Onwuteaka-Philipsen BD. Primary care patients hastening death by voluntarily stopping eating and drinking. Annals of Family Medicine. 2015;13(5):421. 
12. Shinjo $T$, Morita $T$, Kiuchi $D$, et al. Japanese physicians' experiences of terminally ill patients voluntarily stopping eating and drinking: a national survey. BMJ Supportive \& Palliative Care. 2019;9(2):143-5.

13. Ganzini L, Goy ER, Dobscha SK. Why oregon patients request assisted death: Family members' views. Journal of General Internal Medicine. 2008;23(2):154-7.

14. California Department of Public Health. California End of Life Option Act 2018 Data Report. July 2019.

15. Campbell J, Nadel L, Duke D, Ryan L. Remembering all that and then some: recollection of autobiographical memories after a 1-year delay. Memory. 2011;19(4):406-15. 